\title{
SMALL-LARGE SUBGROUPS OF LOCALLY COMPACT ABELIAN POLISH GROUPS
}

\author{
MÁRK POÓR
}

\begin{abstract}
In [1] Rosłanowski and Shelah asked whether every locally compact non-discrete group has a null but non-meager subgroup. In this paper we give an affirmative answer in the Polish Abelian case.
\end{abstract}

\section{INTRODUCTION}

We are interested in null, but non-meager subgroups of locally compact Abelian (LCA) Polish groups. Only the non-discrete case is of interest, since non-empty open sets are of positive measure, thus in a discrete group $\{e\}$ has positive measure, and obviously is of second category. It is known that under the Continuum Hypothesis one can construct null but non-meager and meager but non-null subgroups in the Cantor group or the reals, and Talagrand proved that in the Cantor group there is a null non-meager filter, that is a subgroup too [12. Due to Fremlin and Shelah it is known that an unpublished result of H.M. Friedman implies that in the Cohen model there is no meager but non-null subgroup of the Cantor group [3]. In [11], Rosłanowski, and Shelah constructed $Z F C$ examples for null, but non-meager subgroups of the reals and the Cantor group, and showed that it is consistent with $Z F C$ that in the two groups every meager subgroup is null. Then they asked two questions:

Problem 5.1 [1]

(1) Does every locally compact group (with complete Haar measure) admit a null non-meager subgroup?

(2) Is it consistent that no locally compact group has a meager non-null subgroup?

In the first part of the paper we construct null but non-meager subgroups in every non-discrete locally compact Abelian Polish group. In the second section we will show that it is consistent that in each locally compact Polish group meager subgroups are always null.

The author was supported by the National Research, Development and Innovation Office NKFIH, grants no. 104178, 124749 and 129211.

Supported by the ÚNKP-18-3 New National Excellence Program of the Ministry of Human CaPaCities. 


\section{Preliminaries and notations}

We say that the topological groups $G$ and $H$ are isomorphic, in symbols $G \simeq H$, if there is an algebraic isomorphism, which is also a homeomorphism. Under the symbol $\leq$ we mean the subgroup relation, i.e. $H \leq G$ symbols that $H$ is a subgroup of $G$, and $H \triangleleft G$ is for normal subgroups. All topological groups are assumed to be Hausdorff.

A topological group $G$ is said to be locally compact if for each $g \in G$, there is a neighborhood $B$ of $g$, which is compact (i.e. each point has an open neighborhood which has compact closure). It is known that for a locally compact Hausdorff topological group $G$, if $C \triangleleft G$ is a closed normal subgroup, then $G / C$ is locally compact and Hausdorff. Also, if $G$ is Polish then so is $G / C$. Since in compact Hausdorff spaces the Baire category theorem is true (i.e. no nonempty open set can be covered by countably many nowhere dense sets), it is also true in locally compact Hausdorff spaces.

For any topological space $X, \mathcal{B}(X)$ denotes the Borel sets, i.e. the $\sigma$-algebra generated by the open sets.

Recall the following definition of left Haar measure:

Definition 1. Let $G$ be a locally compact group, and $\mu$ be a Borel measure, i.e.

$$
\mu: \mathcal{B}(G) \rightarrow[0, \infty]
$$

such that

- $\mu$ is left-invariant, i.e. for every $g \in G, B \in \mathcal{B}(G)$

$$
\mu(g B)=\mu(B),
$$

- $\mu(U)>0$ for each open $U \neq \emptyset$,

- $\mu(K)<\infty$ for each compact set $K$,

- $\mu$ is inner regular with respect to the compact sets, that is for every Borel $B$

$$
\mu(B)=\sup \{\mu(K): K \subseteq B, K \text { is compact }\} .
$$

Then $\mu$ is called a left Haar measure of $G$.

It is known that a left Haar measure always exists, and it is unique up to a positive multiplicative constant. By slight abuse of notation we will identify $\mu$ with its completion, i.e. every set $H \subseteq G$ is measurable if $H$ differs from a Borel by (at most) a null Borel set, i.e.

$$
\text { if }\left(\exists B, B^{\prime} \in \mathcal{B}: B \triangle H \subseteq B^{\prime} \wedge \mu\left(B^{\prime}\right)=0\right) \text { then } \mu(H):=\mu(B) \text {. }
$$

Remark 2. There is another definition of (left) Haar measure in locally compact groups, but in locally compact Polish groups (more generally in locally compact groups which are $\sigma$-compact) these two coincide. 
From now on $\mathcal{N}$ will denote the null-ideal w.r.t. a left Haar measure $\mu$, i.e.

$$
\mathcal{N}=\{H \subseteq G: \mu(H)=0\} .
$$

It is known that for any locally compact group the null-ideals of a left Haar measure and a right Haar measure coincide, thus we can speak about null sets ([6, 442F ]).

\section{NULl BUt NON-MEAGER SUBGROUPS}

First, using abstract Fourier analytic methods we will show that the general (Polish LCA) case can be reduced to the case of the circle group, the group of $p$-adic integers, and the direct product of countably many finite (Abelian) groups. Provided that these groups have null but non-meager subgroups, we only have to show that an arbitrary LCA group has a quotient isomorphic to one of the aforementioned three groups, and then verify that pulling-back an appropriate subgroup of the quotient will work. The idea of first finding a nice quotient group, and constructing the desired object for that group is from [5].

Since the direct product of $\omega$-many finite groups, and $\mathbb{R}$ has a null but non-meager subgroup, and so does the circle group by this (see [11]), we only have to construct such subgroups in the group of $p$-adic integers.

The following definition of the $p$-adic integers is from [7, (10.2)].

In Lemma 4 we prove that in any non-discrete LCA group an appropriate open subgroup has a nice quotient group. After that, by Proposition 5 the case of $p$-adic integers is handled.

Finally we summarize, and state our main result in Theorem 7 .

Definition 3. Let $p \in \mathbb{N}$ be a prime. Then consider the product set

$$
\Delta_{p}=\prod_{i=0}^{\infty}\{0,1, \ldots, p-1\}
$$

with the following group operation. Let $x, y \in \Delta_{p}$. Define the series $t \in \prod_{i=0}^{\infty}\{0,1\}$, $z \in \prod_{i=0}^{\infty}\{0,1, \ldots, p-1\}$ inductively so that

$$
x_{0}+y_{0}=t_{0} p+z_{0},
$$

and

$$
t_{i}+x_{i+1}+y_{i+1}=t_{i+1} p+z_{i+1}
$$

holds for each $i \in \omega$. Then $z \in \Delta_{p}$ is the sum of $x$ and $y$.

The topology is defined by the following neighborhood base of 0 (i.e. the identically 0 sequence)

$$
\Lambda_{i}=\left\{x \in \Delta_{p} \mid x_{j}=0 \text { for each } j<i\right\} \quad(i \in \omega) .
$$

Hence the sets of the form

$$
y \Lambda_{n}=\left\{x \in \Delta_{p} \mid x_{j}=y_{j}(j<n)\right\}
$$


is a base consisting of clopen sets. In other words it is the product topology.

Lemma 4. Let $G$ be a locally compact Abelian group, which is non-discrete. Then there is an open subgroup $G^{\prime} \leq G$, and a closed subgroup $C \leq G^{\prime}$ of $G^{\prime}$, such that the quotient group $G^{\prime} / C$ is isomorphic to one of the following:

- the circle group $\mathbb{T} \simeq \mathbb{R} / \mathbb{Z}$,

- the product of $\omega$-many finite (Abelian) groups $\prod_{i \in \omega} G_{i}$ (with each $G_{i}$ having at least two elements),

- the group of p-adic integers $\left(\Delta_{p}\right)$ for some prime $p$.

Proof. By the Principal Structure Theorem of LCA groups [10, 2.4.1], there is an open subgroup $H$ of $G$ of the form $H \simeq K \times \mathbb{R}^{n}$ for some $n \geq 0$, where $K$ is compact. If $n>0$, then $K \times\left(\mathbb{Z} \times \mathbb{R}^{n-1}\right) \triangleleft H$ is a closed normal subgroup of $H$,

$$
H /\left(K \times \mathbb{Z} \times \mathbb{R}^{n-1}\right) \simeq\left(K \times \mathbb{R} \times \mathbb{R}^{n-1}\right) /\left(K \times \mathbb{Z} \times \mathbb{R}^{n-1}\right) \simeq \mathbb{R} / \mathbb{Z} \simeq \mathbb{T},
$$

we are done. Hence we can assume that $n=0$, i.e. $H=K$ is compact. First observe that since $H \leq G$ is open, and $G$ was non-discrete, $H$ cannot be discrete.

Next we claim that the dual group $\hat{H}$ of $H$ is infinite. Assume on the contrary that $\hat{H}$ is finite. Then it is compact, thus the dual $\hat{H}$ of $\hat{H}$ would be discrete (by [10, 1.2.5]). But by the Pontryagin Duality Theorem ([10, 1.7.2]) $\hat{H}$ is isomorphic to $H$ which was not discrete, a contradiction.

Now, by [10, 1.7.2], and [10, 2.1.2] groups of the form $H / C$ (where $C$ is a closed subgroup) are the same (isomorphic as topological groups) as dual groups of the closed subgroups of $\hat{H}$. This means that having a closed subgroup $L \leq \hat{H}$ guarantees that there is a closed subgroup $C \leq H$ such that the quotient group

$$
I=H / C \simeq \hat{L}
$$

(Note that each subgroup of $\hat{H}$ is automatically closed, since according to [10, 1.2.5] the compactness of $H$ implies that $\hat{H}$ is discrete.) Now using [5, Thm. 2.16], and the infiniteness of $\hat{H}$, we obtain that $\hat{H}$ has a subgroup (let's say $L$ ) isomorphic to either

(i) $\mathbb{Z}$, or

(ii) $\oplus_{i \in \omega} G_{i}$ where each $G_{i}$ is a finite group of at least two elements, or

(iii) the quasicyclic group $C_{p^{\infty}}$ for some prime $p$.

This means that

(i) if $L \simeq \mathbb{Z}$, then there is a closed subgroup $C \leq H$ such that

$$
H / C \simeq \hat{L} \simeq \mathbb{T} .
$$

(ii) If $L \simeq \oplus_{i \in \omega} G_{i}$ (where each $G_{i}$ has at least two elements): First note that an LCA group is compact and discrete at the same time iff so is its dual by [10, 1.2.5] and [10, 1.7.2]. This means that an LCA group is finite iff its dual is finite. Second, since the dual of the dual of an LCA group is the group itself [10, 1.7.2], if $\hat{G}_{i}=\{0\}$ was the trivial group, then $G_{i}$ also would be the trivial 
group, therefore each $\hat{G}_{i}$ has at least two elements. From this we obtain that each $\hat{G}_{i}$ is finite, and is of cardinality at least two, and using that the dual of the direct sum (endowed with the discrete topology) is the product of the dual groups [10, 2.2.3] we get that

$$
\left(\oplus_{i \in \omega} G_{i}\right)^{\wedge} \simeq \prod_{i \in \omega} \hat{G}_{i}
$$

Thus there is a closed subgroup $C \leq H$ such that

$$
H / C \simeq \hat{L} \simeq \prod_{i \in \omega} \hat{G}_{i} \quad\left(\forall i:\left|\hat{G}_{i}\right|>1\right) .
$$

(iii) If $L \leq \hat{H}$ is isomorphic to the quasicyclic group $C_{p^{\infty}}$ (for some prime $p$ ), then as $\hat{\Delta_{p}} \simeq C_{p^{\infty}}$ [7, 25.2] we obtain that

$$
\hat{L} \simeq \hat{C}_{p^{\infty}} \simeq \Delta_{p}
$$

by [10, 1.7.2]. Therefore there is a closed subgroup $C \leq H$ for which

$$
H / C \simeq \hat{L} \simeq \Delta_{p}
$$

This shows that there exists a closed subgroup $C \leq H$ such that $I \simeq H / C$ is either the circle group, the $p$-adic integers, or the product of $\omega$-many finite groups, each finite group having at least two elements.

Proposition 5. Let $p \in \mathbb{N}$ be a prime. Then the group of $p$-adic integers has a null but non-meager subgroup.

Proof. Fix a non-principal ultrafilter $\mathcal{U}$ on $\omega$, and for each $j \in \omega, 0 \leq k<(j+1)^{2}-j^{2}$ define the intervals

$$
I_{j}^{k}=\left[j^{2}+k,(j+1)^{2}\right) \subseteq \omega .
$$

Let $\mu$ denote the probability Haar measure (i.e. $\mu\left(\Delta_{p}\right)=1$ ), and

$$
H_{k}=\left\{x \in \Delta_{p} \mid\left\{j \in \omega \mid k<(j+1)^{2}-j^{2} \wedge\left(x_{\mid I_{j}^{k}} \equiv 0 \vee x_{\mid I_{j}^{k}} \equiv p-1\right)\right\} \in \mathcal{U}\right\}
$$

i.e. those which are constant 0 -s or $p-1$-s on $\mathcal{U}$-almost every $I_{j}^{k}$-s. (Note that since $I_{j}^{k} \supseteq I_{j}^{k+1}$ if $j$ is such that both $I_{j}^{k}$ and $I_{j}^{k+1}$ are defined,

$$
\left.H_{k} \subseteq H_{k+1} .\right)
$$

Define the set $H$ as follows

$$
\begin{gathered}
H=\bigcup_{k \in \omega} H_{k}= \\
=\left\{x \in \Delta_{p} \mid \exists k:\left\{j \in \omega \mid(j>(k-1) / 2) \wedge\left(x_{\mid I_{j}^{k}} \equiv 0 \vee x_{\mid I_{j}^{k}} \equiv p-1\right)\right\} \in \mathcal{U}\right\} .
\end{gathered}
$$

We claim that $H$ is the desired subgroup. 
(i) $H$ is a subgroup.

Let $x, y \in H$. Using (6) let $n \in \omega$ be such that $x, y \in H_{n}$. Consider the following elements of $\mathcal{U}$

$$
\begin{aligned}
& U_{x}=\left\{j \in \omega \mid x_{\mid I_{j}^{n}} \equiv 0 \vee x_{\mid I_{j}^{n}} \equiv p-1\right\} \in \mathcal{U}, \\
& U_{y}=\left\{j \in \omega \mid y_{\mid I_{j}^{n}} \equiv 0 \vee y_{\mid I_{j}^{n}} \equiv p-1\right\} \in \mathcal{U},
\end{aligned}
$$

and let $U=U_{x} \cap U_{y} \in \mathcal{U}$ denote their intersection. Now, if $j \in U$, then the following lemma will ensure that $(x+y)_{\mid I_{j}^{n+1}} \equiv 0$, or $(x+y)_{\mid I_{j}^{n+1}} \equiv p-1$, which means that $x+y \in H^{n+1} \subseteq H$.

Lemma 6. Let $x, y \in \Delta_{p}$ and $m<l \in \omega$ such that

$$
x_{\mid[m, l)]} \equiv 0 \text { or } x_{\mid[m, l)]} \equiv p-1
$$

and

$$
y_{\mid[m, l)]} \equiv 0, \text { or } y_{\mid[m, l)]} \equiv p-1
$$

Then

$$
(x+y)_{\mid[m+1, l)]} \equiv 0 \text { or }(x+y)_{\mid[m+1, l)]} \equiv p-1 .
$$

Proof. Let $t \in \prod_{i=0}^{\infty}\{0,1\}, z=x+y \in \prod_{i=0}^{\infty}\{0,1, \ldots, p-1\}$ as in Definition 3. i.e. (11) and (2) hold. Now we have three cases.

- Case 1. If $x_{\mid[m, l)]}=y_{\mid[m, l)]} \equiv 0$, then $x_{m}+y_{m}+t_{m-1}=t_{m-1} \in\{0,1\}$, thus the recursive definition of $t$ and $z$ (2) implies that $t_{m}=0$. From this it is easy to see that $z_{m+1}=z_{m+2}=\cdots=z_{l-1}=0$, i.e. $z_{\mid[m+1, l)]} \equiv 0$, as desired.

- Case 2. If $x_{\mid[m, l)]} \equiv 0$, and $y_{\mid[m, l)]} \equiv p-1$, then $z_{\mid[m+1, l)}$ depends only on $t_{m-1}$. If $t_{m-1}=0$, then

$$
x_{m}+y_{m}+t_{m-1}=p-1=0 \cdot p+p-1,
$$

hence $z_{m}=p-1$, and $t_{m}=0$. Using (2) it is straightforward to check that

$$
t_{m+1}=t_{m+2}=\cdots=t_{l-1}=0,
$$

and

$$
z_{m}=z_{m+1}=\cdots=z_{l-1}=p-1 .
$$

On the other hand, if $t_{m-1}=1$, then

$$
x_{m}+y_{m}+t_{m-1}=p-1+1=1 \cdot p+0,
$$

i.e. $t_{m}=1$, and $z_{m}=0$. Similarly, applying (2) $l-m-1$ times, one can get that

$$
t_{m}=t_{m+1}=t_{m+2}=\cdots=t_{l-1}=1
$$

and

$$
z_{m}=z_{m+1}=\cdots=z_{l-1}=0 .
$$


- Case 3. If $x_{\mid[m, l)]}=y_{\mid[m, l)]} \equiv p-1$,

then

$x_{m}+y_{m}+t_{m-1}=(p-1)+(p-1)+t_{m-1}=1 \cdot p+p-2+t_{m-1}$,

i.e. $t_{m}=1$. Therefore we got that

$$
x_{m+1}+y_{m+1}+t_{m}=(p-1)+(p-1)+1=1 \cdot p+p-1,
$$

i.e. $z_{m+1}=p-1, t_{m+1}=1$. Iterating this argument yields that

$$
t_{m+1}=t_{m+2}=\cdots=t_{l-1}=1,
$$

and

$$
z_{m+1}=z_{m+2}=\cdots=z_{l-1}=p-1 .
$$

It is left to show that $H$ is closed under taking inverse, i.e. if $x \in H_{n}$, then $-x \in H$. We will show that if $i>0$ and then $(-x)_{i}=p-1-x_{i}$ from which we can conclude that

$$
U_{x}=\left\{l \in \omega \mid l>(k-1) / 2 \wedge\left(x_{\mid I_{l}^{n}} \equiv 0 \vee x_{\mid I_{l}^{n}} \equiv p-1\right)\right\} \in \mathcal{U},
$$

then (since $0 \notin I_{l}^{j}$ if $l>0$ )

$$
\begin{aligned}
\{l \in \omega \backslash\{0\} \mid l>(k-1) / 2 & \left.\wedge\left((-x)_{\mid I_{l}^{n}} \equiv 0 \vee(-x)_{\mid I_{l}^{n}} \equiv p-1\right)\right\} \supseteq \\
& \supseteq U_{x} \backslash\{0\} \in \mathcal{U} .
\end{aligned}
$$

Now we have to show that if we define $w \in \Delta_{p}$ so that $w_{0}=p-x_{0}$, $\left.w_{i}=p-1-x_{i}\right)(i>0)$, then $w+x=0$ in $\Delta_{p}$. Let $t \equiv 1 \in \prod_{i=0}^{\infty}\{0,1\}$ the constant 1 function. Now it is easy to see that $x_{0}+w_{0}=t_{0} p$ and $x_{i}+w_{i}+1=t_{i} p$, thus according to the rule of addition in $\Delta_{p}$, (11), (2) ), $x+w=0$, i.e. $w=-x$.

(ii) $H$ is null.

Since

$$
H=\bigcup_{k \in \omega} H_{k}
$$

it is enough to show that $\mu\left(H_{k}\right)=0$ for each $k$. But using the non-principality of $\mathcal{U}$

$$
\begin{gathered}
\mu\left(H_{k}\right)= \\
=\mu\left(\left\{x \in \Delta_{p} \mid\left\{j \in \omega \mid j>(k-1) / 2 \wedge\left(x_{\mid I_{j}^{k}} \equiv 0 \vee x_{\mid I_{j}^{k}} \equiv p-1\right)\right\} \in \mathcal{U}\right\}\right) \leq \\
\leq \mu\left(\left\{x \in \Delta_{p}||\left\{j \in \omega \mid j>(k-1) / 2 \wedge\left(x_{\mid I_{j}^{k}} \equiv 0 \vee x_{\mid I_{j}^{k}} \equiv p-1\right)\right\} \mid=\infty\right\}\right)
\end{gathered}
$$

i.e. it suffices to show that those sequences, for which there exist infinitely many $j$-s such that the sequence is constant 0 or $p-1$ on $I_{j}^{k}$ form a null set. Now, we know that for each $m$ the $p^{m}$-many translates of the open set

$$
\Lambda_{m}=\left\{x \in \Delta_{p} \mid x_{l}=0 \text { for each } l<m\right\}
$$


is a partition of $\Delta_{p}$ (3), therefore because $\mu$ is a translation-invariant probability measure

$$
\mu\left(y \Lambda_{m}\right)=\mu\left(\left\{x \in \Delta_{p} \mid x_{l}=y_{l}(\forall l<m)\right\}\right)=\frac{1}{p^{m}},
$$

hence

$\mu\left(\left\{x \in \Delta_{p} \mid x_{I_{j}^{k}} \equiv 0\right\}\right)=\mu\left(\left\{x \in \Delta_{p} \mid x_{I_{j}^{k}} \equiv p-1\right\}\right)=\frac{1}{p^{\left|I_{j}^{k}\right|}}=\frac{1}{p^{2 j+1-k}}$.

Since this gives us that

$$
\mu\left(\left\{x \in \Delta_{p} \mid x_{I_{j}^{k}} \equiv 0 \vee x_{I_{j}^{k}} \equiv p-1\right\}\right)=\frac{2}{p^{2 j+1-k}},
$$

for arbitrary fixed $l_{0}$ the following inequality will hold

$$
\begin{gathered}
\mu\left(\left\{x \in \Delta_{p}||\left\{j \in \omega \mid j>(k-1) / 2 \wedge\left(x_{\mid I_{j}^{k}} \equiv 0 \vee x_{\mid I_{j}^{k}} \equiv p-1\right)\right\} \mid=\infty\right\}\right)= \\
=\mu\left(\bigcap_{l=0}^{\infty} \bigcup_{j=l}^{\infty}\left\{x \in \Delta_{p} \mid\left(x_{\mid I_{j}^{k}} \equiv 0 \vee x_{\mid I_{j}^{k}} \equiv p-1\right)\right\}\right) \leq \\
\leq \mu\left(\bigcup_{j=l_{0}}^{\infty}\left\{x \in \Delta_{p} \mid\left(x_{\mid I_{j}^{k}} \equiv 0 \vee x_{\mid I_{j}^{k}} \equiv p-1\right)\right\}\right) .
\end{gathered}
$$

We obtained the following obvious upper bound

$$
\mu\left(\bigcup_{j=l_{0}}^{\infty}\left\{x \in \Delta_{p} \mid\left(x_{\mid I_{j}^{k}} \equiv 0 \vee x_{\mid I_{j}^{k}} \equiv p-1\right)\right\}\right) \leq \sum_{j=l_{0}}^{\infty} \frac{2}{p^{2 j+1-k}},
$$

and as the latter tends to 0 when $l_{0}$ tends to infinite, we are done.

(iii) $H$ is non-meager.

We will show that even $H_{0}$ is non-meager. For each $j \in \omega$ define the mapping $f_{j}$ as follows

$$
\begin{gathered}
f_{j}:\{0,1, \ldots p-1\}^{\left|I_{j}^{0}\right|} \rightarrow\{0,1\} \\
\underline{v} \mapsto\left\{\begin{array}{cc}
0 & \text { iff } v \equiv 0 \\
1 & \text { otherwise }
\end{array}\right.
\end{gathered}
$$

Let $f$ be the following mapping from $\Delta_{p}$ to $2^{\omega}$ (where we identify $\Delta_{p}$ with $\left.\{0,1, \ldots p-1\}^{\omega}\right)$

$$
\begin{gathered}
f: \Delta_{p} \rightarrow 2^{\omega} \\
x \mapsto\left(f_{j}\left(x_{\mid I_{j}^{0}}\right)\right)_{j \in \omega}
\end{gathered}
$$

Now suppose that $\Delta_{p} \backslash H$ is co-meager. As $f$ is a continuous surjective open mapping between Polish spaces, according to [1, Lemma 2.6] $f$ maps a comeager set onto a co-meager set, let $R=f\left(\Delta_{p} \backslash H\right)$ denote this co-meager set in $2^{\omega}$. Now, using [2, Thm. 2.2.4 ]), there exist a strictly growing sequence of non-negative integers

$$
0=n_{0}<n_{1}<\ldots
$$


and an element $r \in 2^{\omega}$ such that

$$
\left\{s \in 2^{\omega}||\left\{j: s_{\mid\left[n_{j}, n_{j+1}\right)} \equiv r_{\mid\left[n_{j}, n_{j+1}\right)}\right\} \mid=\infty\right\} \subseteq R .
$$

Now the following disjoint sets cover $\omega$ (since the sequence $n_{j}$ is strictly growing)

$$
U_{0}=\bigcup_{k \in \omega}\left[n_{2 k}, n_{2 k+1}\right), \quad U_{1}=\bigcup_{k \in \omega}\left[n_{2 k+1}, n_{2 k+2}\right),
$$

thus exactly one of them is in $\mathcal{U}$. Let $U$ denote that set. Now define the following element $y$ of $2^{\omega}$

$$
y_{i}=\left\{\begin{array}{cc}
0 & \text { iff } i \in U \\
r_{i} & \text { otherwise }
\end{array}\right.
$$

Clearly (using (10) and the fact that $U=U_{i}$ for some $i \in\{0,1\}$ ) there are infinitely many $j$-s for which $y_{\mid\left[n_{j}, n_{j+1}\right)} \equiv r_{\mid\left[n_{j}, n_{j+1}\right)}$, thus by (99)

$$
y \in R=f\left(\Delta_{p} \backslash H\right)
$$

Hence, there is a

$$
z \in \Delta_{p} \backslash H
$$

for which

$$
f(z)=y
$$

But for each $j \in \omega$, if

$$
j \in U
$$

$\Downarrow$ (by (8) and (11))

$$
\begin{gathered}
y_{j}=f_{j}\left(z_{\mid I_{j}^{0}}\right)=0 \\
\Downarrow(\text { by (77) }) \\
z_{\mid\left[j^{2},(j+1)^{2}\right)} \equiv 0 .
\end{gathered}
$$

This means, as $U \in \mathcal{U}$, that we found that for $\mathcal{U}$-almost every $j, z_{\mid\left[j^{2},(j+1)^{2}\right)} \equiv 0$, therefore $z \in H_{0}$ by the definition of $H_{0}$ (5), contradicting (12).

Now we are ready to state our result.

Theorem 7. Let $G$ be a non-discrete Polish LCA group. Then there is a null, non-meager subgroup in $G$.

Proof. According to Lemma 4 there is an open subgroup $G^{\prime} \leq G$ for which there exists a closed normal subgroup $C \triangleleft G^{\prime}$ such that $G^{\prime} / C$ is isomorphic to one of the following

(i) the circle group $\mathbb{T} \simeq \mathbb{R} / \mathbb{Z}$,

(ii) the product of $\omega$-many finite (Abelian) groups $\prod_{i \in \omega} G_{i}$ (with each $G_{i}$ having at least two elements),

(iii) the group of $p$-adic integers $\left(\Delta_{p}\right)$.

First we show that 
Claim 8. $G^{\prime} / C$ has a null but non-meager subgroup, say $H / C$ (where $C \leq H \leq$ $\left.G^{\prime}\right)$.

Proof. In case (i), first, consider the null non-meager subgroup $L \leq \mathbb{R}$ given by [11, Theorem 2.3]. Recall that the Haar measure is the Lebesgue measure on the real line (up to a positive multiplicative constant). After identifying the circle group with $[0,1)$, the Haar measure on it coincides with the Lebesgue measure restricted to the unit interval. Therefore the set

$$
L^{\prime}=\{r \in[0,1): \exists k \in \mathbb{Z} \quad k+r \in L\} \subseteq[0,1)=\mathbb{T}
$$

is a null set, and forms a subgroup in the circle group. $L^{\prime} \subseteq[0,1)$ is non-meager, since the meagerness of $L^{\prime}$ would imply that $L^{\prime}+\mathbb{Z} \supseteq L$ was meager in $\mathbb{R}$. Given this null and non-meager subgroup $L^{\prime} \leq \mathbb{T} \simeq G^{\prime} / C$, let $H \leq G^{\prime}$ be such that $H / C \leq G^{\prime} / C \simeq \mathbb{T}$ is a null, but nonmeager subgroup.

In case (iii), i.e.

$$
G^{\prime} / C \simeq \prod_{i \in \omega} F_{i} \quad\left(\forall i: 1<\left|F_{i}\right|<\infty\right)
$$

11, Remark 4.2] implies that there is a null but non-meager subgroup in $G^{\prime} / C$, say $H / C$.

If $G^{\prime} / C$ is isomorphic to the group of the $p$-adic integers for some prime $p$, then Proposition 5 guarantees that there is an appropriate subgroup in $G^{\prime} / C$.

Next we have to verify that from the nullness and non-meagerness of $H / C$ (in $G^{\prime} / C$ ) follows the nullness and non-meagerness of $H$ in $G^{\prime}$. Let $\pi: G^{\prime} \rightarrow G^{\prime} / C$ denote the canonical projection. Now since a Haar measure $\nu$ on $G^{\prime} / C$ is also $G^{\prime}$-invariant and Radon, using [6, 443P/(c)] (with $X=G^{\prime}, Y=C, \lambda=\nu$ ) we obtain that $H \leq G^{\prime}$ is a subgroup of measure zero.

For the non-meagerness, suppose that $G^{\prime} \backslash H$ is co-meager in $G^{\prime}$. Then using 11. Lemma 2.6] (and the fact $C \leq H$ ), the image of $G^{\prime} \backslash H$ under the canonical projection $\pi: G^{\prime} \rightarrow G^{\prime} / C$

$$
\pi\left(G^{\prime} \backslash H\right)=\left(G^{\prime} / C\right) \backslash(H / C)
$$

would also be co-meager (in $G^{\prime} / C$ ), contradicting that $H / C$ is non-meager.

Now, we have a null but non-meager subgroup $H$ in $G^{\prime}$, but since $G^{\prime} \leq G$ is open, it is straightforward to check that a Haar measure of $G$ restricted to $G^{\prime}$ is a Haar measure of $G^{\prime}$, therefore $H$ is null in $G$ too. Moreover a non-meager set in an open subspace (namely $G^{\prime}$ ) is also non-meager in the whole space, thus $H \leq G$ is a null non-meager subgroup. 


\section{Meager BUt NON-NULl SUBGROUPS}

The following result can be found in [9], but for the sake of completeness we include the sketch of the proofs. We will prove that it is consistent with $Z F C$ that in every locally compact Polish group meager subgroups are null.

We will use H. M. Friedman's theorem, which can be found in [3]:

Theorem 9 (H. M. Friedman). In the Cohen model (that is, adding $\omega_{2}$ Cohen reals to a model of $Z F C+C H$ ) the following holds:

$$
\begin{aligned}
& \forall H \subseteq 2^{\omega} \times 2^{\omega} F_{\sigma}: \\
& \text { if }(\exists C \times D \subseteq H, C \times D \notin \mathcal{N}) \text {, then }(\exists A \times B \subseteq H, A \times B \notin \mathcal{N} \text { measurable })
\end{aligned}
$$

This property of $2^{\omega}$ implies that every meager subgroup of $2^{\omega}$ is of measure zero. Similarly if it holds in a locally compact Polish group $G$, then $G$ has no meager non-null subgroups, this lemma is also from [3] (stating it only for $G=2^{\omega}$, but the proof is the same).

Lemma 10. Let $G$ be a locally compact Polish group, $\mu$ be a left Haar measure on $G$. Assume that the following holds:

$$
\begin{aligned}
& \forall H \subseteq G \times G, F_{\sigma}: \\
& \left(\exists C \times D \subseteq H, C \times D \notin \mathcal{N}_{\mu}\right) \longrightarrow\left(\exists A \times B \subseteq H, A \times B \notin \mathcal{N}_{\mu} \text { measurable }\right)
\end{aligned}
$$

Then every meager subgroup of $G$ is null.

Remark 11. If $X$ is a Polish space, $\nu$ is a $\sigma$-finite Borel measure on $X$, then for every $\nu$-measurable set $H$ there exists a Borel $B$ such that $H \triangle B$ is null (wrt. $\nu$ ).

In Lemma 12 we show that if the condition of Lemma 10 holds in $2^{\omega}$ (e.g. in the Cohen model), then this holds for arbitrary locally compact Polish groups. This yields that it is consistent with $Z F C$ that in locally compact Polish groups meager subgroups are always null.

Lemma 12. Assume that the condition from Lemma 10 holds in $2^{\omega}$ (i.e. every $F_{\sigma}$ subset of $2^{\omega} \times 2^{\omega}$ which contains a non-null rectangle must contain a measurable non-null rectangle). Then this condition holds in every locally compact Polish group $G$.

Combining Theorem 9 with Lemma 10 yields the following.

Theorem 13. It is consistent with ZFC that in every locally compact Polish group meager subgroups are always null.

Remark 14. If $X$ is a Polish space, and $\mu$ is a $\sigma$-finite Borel measure, then for any rectangle $C \times D \subseteq X \times X$, it is non-null (wrt. the product measure $\mu \times \mu$ ) iff $C \notin \mathcal{N}_{\mu}$ and $D \notin \mathcal{N}_{\mu}$.

Proof. (Lemma 12) Assume that $\mu$ denotes the left Haar measure on $G, \nu$ denotes the Haar measure on $2^{\omega}$. Then since locally compact Polish groups are $\sigma$-compact, the Haar measure $\mu$ is $\sigma$-finite. 
Lemma 15. There exist $F_{\sigma}$ sets $C \subseteq 2^{\omega}$ and $K \subseteq G$ of full measure, and a Borel bijection $f: C \rightarrow K$ such that for each $S \subseteq C$

(i) $\nu(S)=0$ iff $\mu(f(S))=0$,

(ii) $S$ is measurable iff $f(S)$ is measurable,

and for the bijection $f \times f: C \times C \rightarrow K \times K$

$$
S^{\prime} \subseteq C \times C \text { is } F_{\sigma} \quad \text { iff } \quad(f \times f)\left(S^{\prime}\right) \subseteq K \times K \text { is } F_{\sigma} .
$$

Proof. For the construction of $f$ we will use the following claim. One can prove it using the regularity of $\nu$ and $\mu$, the isomorphism theorem for measures [8, Thm 17.41.] and Lusin's theorem (see [8, Thm 17.12]).

Claim 16. There is a sequence of pairwise disjoint compact sets $C_{i}(i \in \omega)$ in $2^{\omega}$, for which $\nu\left(C_{i}\right)>0$ and $\nu\left(2^{\omega} \backslash \bigcup_{i \in \omega} C_{i}\right)=0$, and similarly a sequence of pairwise disjoint compact sets $K_{i}(i \in \omega)$ in $G$ with $\mu\left(K_{i}\right)>0$ and $\mu\left(G \backslash \bigcup_{i \in \omega} K_{i}\right)=0$, and there exist homeomorphisms $f_{i}: C_{i} \rightarrow K_{i}$, and positive constants $r_{i}$ such that

$$
\forall B \subseteq C_{i} \text { Borel: } \nu(B)=r_{i} \mu\left(f_{i}(B)\right) .
$$

Now let $C=\bigcup_{i \in \omega} C_{i} \subseteq 2^{\omega}, K=\bigcup_{i \in \omega} K_{i} \subseteq G, f=\bigcup_{i \in \omega} f_{i}$ given by Claim 16 , Then

$$
f: C \rightarrow K
$$

is a Borel bijection, and $C, K$ and $f$ satisfy conditions (ii) - (iii) (by Remark [11, $S$ differs from a Borel by a null set, $f^{-1}$ is a Borel function, and $f$ maps a null set to a null set). For (15) note that $f \times f=\bigcup_{i, j \in \omega} f_{i} \times f_{j}$ is the union of homeomorphisms between compact sets.

Observe that $K \times K \subseteq G \times G, C \times C \subseteq 2^{\omega} \times 2^{\omega}$ are $F_{\sigma}$ sets of full measure.

Fix an $F_{\sigma}$ set $F \subseteq G \times G$, and let $D \times E \subseteq F$ be a non-null rectangle. Since by Remark $14 D$ and $E$ are non-null sets and $\mu(G \backslash K)=0,(D \cap K) \times(E \cap K)$ is a non-null rectangle. Moreover, since $K \times K$ is an $F_{\sigma}$ set of full measure (w.r.t. $\mu \times \mu)$

$$
(D \cap K) \times(E \cap K) \subseteq F \cap(K \times K),
$$

i.e. the $F_{\sigma}$ set $F \cap(K \times K)$ contains a non-null rectangle, therefore we can assume that $F \subseteq K \times K$, it suffices to find measurable non-null rectangles in such $F$-s.

If $D \times E \subseteq F$ is a non-null rectangle, then $f^{-1}(D) \times f^{-1}(E)$ is non-null by (ii). Now since $(f \times f)^{-1}(F) \subseteq 2^{\omega} \times 2^{\omega}$ is $F_{\sigma}$ (by (15)) containing the non-null rectangle $f^{-1}(D) \times f^{-1}(E)$, it also contains a product $A \times B$ with $A, B$ measurable, $\nu(A), \nu(B)>0$. Then by (ii), (iii) $f^{-1}(A), f^{-1}(B)$ are measurable, $\mu$-positive subsets of $G$ such that $f^{-1}(A) \times f^{-1}(B) \subseteq F$, as desired. 
In [9] we gave affirmative answers to both questions from [11, Problem 5.1] in the general (locally compact, not necessarily Polish) case. However, due to Christensen we can extend the notion of the null ideal to every (not necessarily locally compact) Polish group. We say that a subset $X$ of a Polish group is Haar-null (in the sense of Christensen), if there is a Borel probability measure $\mu$ on $G$, and a Borel set $B \supseteq X$, such that for each $g, h \in G \mu(g B h)=0$ [4. The following question is open.

Question 17. What can we say about small-large subgroups of non-locally compact Polish groups, replacing "null wrt. the Haar measure" by "Haar-null"? Do (always) exist Haar-null but non-meager, and meager but non-Haar-null subgroups in nonlocally compact Polish groups?

\section{REFERENCES}

[1] Balka, R., Buczolich, Z. and Elekes, M.: Topological Hausdorff dimension and level sets of generic continuous functions on fractals. Chaos Solutions Fractals 45, pp 1579-1589., 2012.

[2] Bartoszyński, T., Judah, H: Set Theory. On the Structure of the Real Line. A K Peters, 1995.

[3] Burke, M. R.: A theorem of Friedman on rectangle inclusion and its consequences. Note of March 7, 1991.

[4] Christensen, J.P.R.: On sets of Haar measure zero in abelian Polish groups. Israel J. Math. 13, pp 255-260., 1972.

[5] Elekes, M., Tóth, Á.: Covering locally compact groups by less than $2^{\omega}$ many translates of a compact nullset. Fund. Math. 193, pp 243-257., 2007.

[6] Fremlin, D. H.: Measure Theory: Volume 4, Topological Measure Spaces 2003.

[7] Hewitt, E., Ross, K.A.: Abstract harmonic analysis. Vol I., 2nd ed, Springer, 1979.

[8] Kechris, A. S.: Classical Descriptive Set Theory. Graduate Texts in Mathematics, 156, Springer-Verlag, 1995.

[9] Poór, M.: Answer to a question of Rostanowski and Shelah. arXiv: 1610.00614, 2016.

[10] Rudin, W.: Fourier analysis on groups. John Wiley \& Sons, 1990.

[11] Rosłanowski, A., Shelah, S.: Small-large subgroups of the reals. Mathematica Slovaca 68, Issue 3, pp 473-484., 2018.

[12] Talagrand M.: Compacts de fonctions mesurables et filtres non mesurables. Studia Math. 67, pp 13-43., 1980

Eötvös Loránd University, Institute of Mathematics, Pázmány Péter s. 1/C, 1117 BuDAPESt, Hungary

E-mail address: sokmark@caesar.elte.hu 